\title{
Properties and Applications of the Simplified Generalized Perpendicular Bisector
}

\author{
Aurélie Richard, Gaëlle Largeteau-Skapin, Marc Rodríguez, Eric Andres, \\ Laurent Fuchs, and Jean-Serge Dimitri Ouattara \\ Laboratory XLIM, SIC Department, \\ University of Poitiers BP 30179, UMR CNRS 6712 \\ 86962 Futuroscope Chasseneuil Cedex, France
}

\begin{abstract}
This paper deals with the Simplified Generalized Perpendicular Bisector (SGBP) presented in 15]1. The SGPB has some interesting properties that we explore. We show in particular that the SGPB can be used for the recognition and exhaustive parameter estimation of noisy discrete circles. A second application we are considering is the error estimation for a class of rotation reconstruction algorithms.
\end{abstract}

Keywords: Simplified Generalized Perpendicular Bisector - Adaptive Pixel Size - Generalized Reflection Symmetry - Rotation Reconstruction.

\section{Introduction}

In this paper we are discussing properties and applications of the Simplified Generalized Perpendicular Bisector (SGPB) that has been introduced in 151]. The SGPB is an extension of the classical notion of perpendicular bisector. The Generalized Perpendicular Bisector (GPB) for two 2D regions $A$ and $B$ is defined as the union of the perpendicular bisectors of all the couple of points $(p, q)$ where $p$ and $q$ are respectively points of the regions $A$ and $B$. The regions we are going to focus on are pixels. The boundary of a GPB between two pixels is composed of line segments and parabola segments. In order to simplify the computational aspects, the SGPB has been introduced. It is only composed of straight line segments. Contrary to a $2 \mathrm{D}$ perpendicular bisector, the GPB and SGPB are not lines but surfaces. Bisectors between points and curves or between two curves have also been discussed in detail in the literature [7]1] but to our best knowledge, no definition for the bisector between two coplanar surfaces such as pixels has been proposed before [15].

The SGPB has some interesting properties and can be useful in several different applications mainly when noisy data is considered. The first application we present is the adaptive pixel SGPB and its use for noisy circle recognition. This is similar to the idea presented in 14 for discrete straight lines. The second application concerns the parameter estimation of rotations. For this, the Generalized Perpendicular Symmetry transform is introduced. We use this to examine the rotation estimation algorithm recently proposed by Fontjine et al. 8] and illustrate its behaviour for noisy data. 


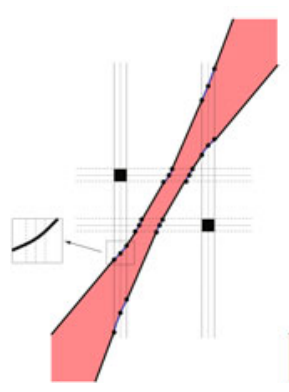

(a)

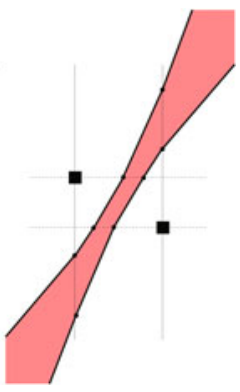

(b)

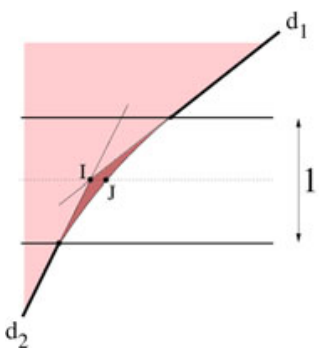

(c)

Fig. 1. The exact perpendicular bisector (a) and the approximation (b) where the parabolic pieces have been dropped by extending the straight lines (c). The generalized bisector is slightly reduced.

The paper is organized as follows: section 2 recalls some properties of the SGPB. The section 3 is devoted to the SGBP and the adaptive pixel size SGBP. In particular some of its properties are highlighted and an application to noisy circle recognition is provided. The next section deals with the symmetry relatively to a SGPB and its application to rotation parameter estimation.

\section{Definition and Properties of the SGBP [15, 1]}

As mentioned in the introduction, the Generalized Perpendicular Bisector (GPB) between two 2D regions $A$ and $B$ (see Figure 1(a)) is defined as the union of the perpendicular bisectors of all the couples of points $(p, q)$ where $p$ (resp. $q$ ) belongs to the regions $A$ (resp. $B$ ). In our case we are considering bounded connected regions since we focus on regions that are pixels (that may have different sizes).

By definition, points of the perpendicular bisector are equidistant to both points $p$ and $q$. Thus a particular point $r$ on the perpendicular bisector is the center of a hypersphere that passes through $p$ and $q$. Hence, we can set an alternative definition of the GPB. Let $\mathcal{S}_{1}$ and $\mathcal{S}_{2}$ be two bounded connected regions and $X$ an Euclidean point of $\mathbb{R}^{n}$. Let $d_{i_{\text {min }}}(X)=\min _{Y \in \mathcal{S}_{i}}(d(X, Y))$, $d_{i_{\text {max }}}(X)=\max _{Y \in \mathcal{S}_{i}}(d(X, Y))$ where $d$ is the usual Euclidean distance. Every Euclidean point $X \in \mathbb{R}^{n}$ such that:

$$
\left[d_{1_{\min }}(X), d_{1_{\max }}(X)\right] \bigcap\left[d_{2_{\min }}(X), d_{2_{\max }}(X)\right] \neq \emptyset
$$

belongs to the GPB of $\mathcal{S}_{1}$ and $\mathcal{S}_{2}$ (see Figure 1(a)). The boundary of the GPB between two pixels is composed of line segments and parabola segments. The parabola segments can be easily removed by extending the line segments. This defines the Simplified GPB (SGPB) (See Figure1(b,c)). Another way of considering this is simply to state that the minimum distance to a pixel is approximated by the distance to the closest vertex of the pixel rather than to the closest vertex or edge. In other words, the SGPB of two pixels $P_{1}$ and $P_{2}$ of size respectively $\lambda_{1}$ and $\lambda_{2}$ is given by the following equations: 


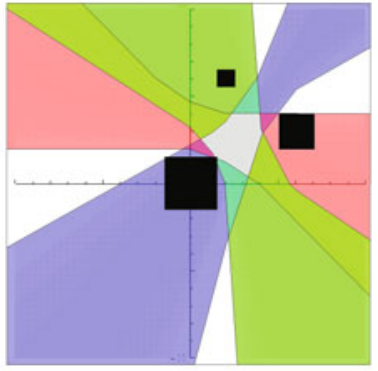

(a)

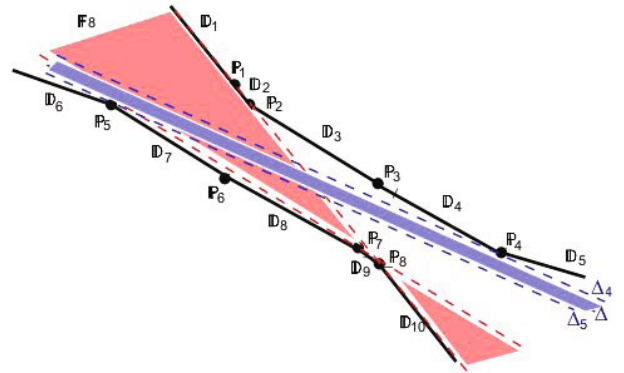

(b)

Fig. 2. (a) Simplified Generalized Perpendicular Bisector and Simplified Generalized Circumcenter of three adaptive pixels ; (b) The 6 border segments and the 4 half-lines of a SGPB $\left\{\mathbb{D}_{i}\right\}_{i \in[1,10]}$ and its characteristic points $\left\{\mathbb{P}_{i}\right\}_{i \in[1,8]}\left(\mathbb{D}_{1}=\mathbb{D}_{10}\right.$ and $\left.\mathbb{D}_{5}=\mathbb{D}_{6}\right)$. The line bundle $\mathbb{F}_{8}$ and the line beam $\operatorname{beam}\left(\mathbb{P}_{4}, \mathbb{P}_{5}\right)$.

$$
\begin{aligned}
& \operatorname{SGPB}\left(P_{1}, P_{2}\right)=\left\{(x, y) \in \mathbb{R}^{2},\right. \\
& \left(\sqrt{\left(x-C_{2 x}\right)^{2}+\left(y-C_{2 y}\right)^{2}} \leq \sqrt{\left(x-F_{1 x}\right)^{2}+\left(y-F_{1 y}\right)^{2}}\right) \\
& \left.\wedge\left(\sqrt{\left(x-C_{1 x}\right)^{2}+\left(y-C_{1 y}\right)^{2}} \leq \sqrt{\left(x-F_{2 x}\right)^{2}+\left(y-F_{2 y}\right)^{2}}\right)\right\}
\end{aligned}
$$

where $C_{i x}, F_{i x} \in\left\{\left(x_{i}+\frac{\lambda_{\mathbf{i}}}{2}\right),\left(x_{i}-\frac{\lambda_{\mathbf{i}}}{2}\right)\right\}, C_{i y}, F_{i y} \in\left\{\left(y_{i}+\frac{\lambda_{\mathbf{i}}}{2}\right),\left(y_{i}-\frac{\lambda_{\mathbf{i}}}{2}\right)\right\}$.

The SGPB between two pixels $P_{1}$ and $P_{2}$ is bounded by line segments and half-lines as shown in the Figure 2(b).

Proposition 1. The boundary of 2D-Simplified Generalized Perpendicular Bisector between two pixels $\left(x_{1}, y_{1}\right)$ of size $\lambda_{1}$ and $\left(x_{2}, y_{2}\right)$ of size $\lambda_{2}$ is composed by at most 10 line segments and half-lines.

The proof of this proposition is straightforward.

Let us introduce some concepts and notations useful for the purpose of this section. Let $\left\{\mathbb{D}_{i}\right\}_{i \in[1,10]}$ be the border segments and half-lines of $\operatorname{SGPB}\left(P_{1}, P_{2}\right)$. Let $\left\{\mathbb{P}_{i}\right\}_{i \in[1,8]}$ be the characteristic points of $S G P B\left(P_{1}, P_{2}\right)$ : i.e. $\mathbb{P}_{i}$ belongs to the border of the $\operatorname{SGPB}\left(P_{1}, P_{2}\right)$ and we organize in such a way that $\mathbb{P}_{i}=\mathbb{D}_{i} \cap$ $\mathbb{D}_{i+1}$ if $i \in[1 ; 4]$ and $\mathbb{P}_{i}=\mathbb{D}_{i+1} \cap \mathbb{D}_{i+2}$ if $i \in[5 ; 8]$ (see Figure[2(b)). $\mathbb{D}_{1}, \mathbb{D}_{10}, \mathbb{D}_{5}, \mathbb{D}_{6}$ are the support lines for the half-lines on the border of the $\operatorname{SGPB}\left(P_{1}, P_{2}\right)$. In fact, as the following lemma shows half-lines are linked two by two:

A first immediate property is given by following lemma:

Lemma 1. Let $A\left(a_{1}, a_{2}\right)$ of size $\lambda_{A}$ and $B\left(b_{1}, b_{2}\right)$ of size $\lambda_{B}$ be two pixels such as $a_{i} \pm \frac{\lambda_{A}}{2} \neq b_{i} \pm \frac{\lambda_{B}}{2}$. The border segments $\mathbb{D}_{1}$ and $\mathbb{D}_{10}$ are supported by the same line and this is also the case for segments $\mathbb{D}_{5}$ and $\mathbb{D}_{6}$.

The proof of this lemma is straightforward using formula (1). It is also easy to see that in case $a_{i} \pm \frac{\lambda_{A}}{2}=b_{i} \pm \frac{\lambda_{B}}{2}$ then $\mathbb{D}_{1}$ and $\mathbb{D}_{10}$ or $\mathbb{D}_{5}$ and $\mathbb{D}_{6}$ are not equal but parallel. 


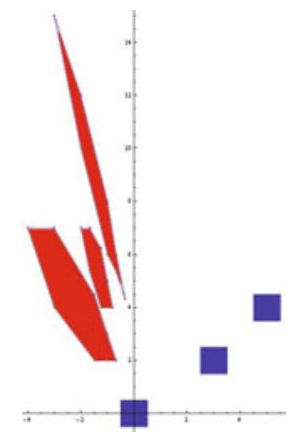

Fig. 3. Dual of three SGPB corresponding to three pixels

\subsection{Dual of a SGPB}

It is easy to determine if a point belongs to a SGPB but not so for a straight line although the SGPB is defined as a union of straight lines. One way of handling this problem is to consider the dual of a SGPB. The dual (see [6] for more details) of the perpendicular bisector between Euclidean points $A\left(x_{a}, y_{a}\right)$ and $B\left(x_{b}, y_{b}\right)$ is defined as the point $\left(\frac{x_{b}-x_{a}}{y_{a}-y_{b}}, \frac{x_{a}^{2}-x_{b}^{2}+y_{a}^{2}-y_{b}^{2}}{2\left(y_{a}-y_{b}\right)}\right)$ for $y_{A}-y_{B} \neq 0$. In the case where $y_{A}-y_{B}=0$ we have a point at the infinity.

Proposition 2. The dual of a $S G P B$ is a convex polygon of at most 8 vertices and 8 edges. At most two vertices may be at the infinite and the corresponding edges of the dual polygon are in this case vertical.

Proof. The boundary of the SGPB is formed of straight lines segments and halflines. The dual is therefore a polygon. It is easy to see that this polygon is convex. Let us consider two points $A$ and $B$ on the boundary of the dual of a SGPB. These two points correspond to two straight lines $L_{A}$ and $L_{B}$ that belong to the SGPB. The intersection point $I$ between $L_{A}$ and $L_{B}$ corresponds to the straight line $A B$ in the dual. The point $I$ is inside the SGPB. All the straight lines passing through $I$ form two bundles delimited by $L_{A}$ and $L_{B}$ with one of them containing only lines that are inside the SGPB since $L_{A}$ and $L_{B}$ are inside the SGPB. The other bundle contains lines that are outside the SGPB. A point outside the segment $[A B]$ and not inside the polygon corresponds to a straight line that does not belong to the SGPB and thus to the bundle that contains lines that do not belong to the SGPB. The points that belong to the segment $[A B]$ correspond therefore to straight lines of the bundle that contain only lines inside the SGPB. All the points of $[A B]$ are therefore inside the polygon and this proves that the polygon is convex. The number of vertices and edges of the polygon is an immediate result of Proposition 1.

Determining if a point belongs to a convex polygon with bounded number of edges and thus if a straight line belongs to the SGPB is trivially done in $O(1)$. 


\section{Adaptive Pixel SGPB and Noisy Circle Recognition}

In [14] some of the authors considered a straight line recognition method that takes into account noise by locally increasing the size of the various pixels. Using a resizing function 14 and a local noise estimator (for example the one proposed by Kerautret et al. [10]), pixel sizes are adaptively increased according to the local perturbation on the straight line. The size increased pixels are called Adaptive Pixels 14. The idea here is to do exactly the same with the SGPB for noisy circle recognition purposes.

First, let us introduce the Simplified Generalized Circumcenter $(S G C)$ of a set of $n$ finite and connected regions $\mathcal{S}=\left(S_{i}\right)_{i \in[1, n]}$. It is defined as the intersection of the SGPB of every two regions of the set (see Figure 2(a)) :

$$
S G C(\mathcal{S})=\bigcap_{i, j \in[1, n], i<j}\left(\operatorname{SGPB}\left(S_{i}, S_{j}\right)\right) .
$$

Theorem 1. Each point of the Simplified Generalized Circumcenter corresponds to the center of at least one circle that intersects all the Adaptive Pixels.

Proof. The proof is similar to the one of Theorem 1 presented in [15]: Each point of the $S G C$ obtained by the intersection of adaptive pixel $S G B P$ s corresponds to an intersection of radii intervals (dimension 1) for every two adaptive pixels. A direct application of Helly's Theorem tell us that there exists at least one common radius to all these intervals and thus at least one circle of this radius centered on the $S G C$ point that intersects all the adaptive pixels.

Proposition 3. Let us consider a set of adaptive pixels $P_{i}$ of various sizes. The dual of all the straight lines crossing the duals of all the SGPB of every pair of pixels $P_{i}$ and $P_{j}$ is the dual of the Simplified Generalized Circumcenter.

This proposition is an immediate consequence of the definition of the $S G C$ and the definition of our notion of dual.

\subsection{Application to Noisy Circle Recognition}

On the Figure 4, we can see a Bresenham circle of radius 5 with misplaced and missing pixels. The $S G C$ can be seen in the middle of the different adaptive pixels. One circle example (the center is marked by a black dot in the $S G C$ ) is shown. Each pixel size is increased according to a local noise estimator. The algorithm is the same as the one for regular circles presented in [15]. The SGPB of each couple of pixels (with the new sizes) is computed and their intersection provides a set of possible circle centers, the Simplified Generalized Circumcenter $(S G C)$. A further computation provides for each point of the $S G C$, the interval of possible circle radii that correspond to circles intersecting all the size increased pixels. 


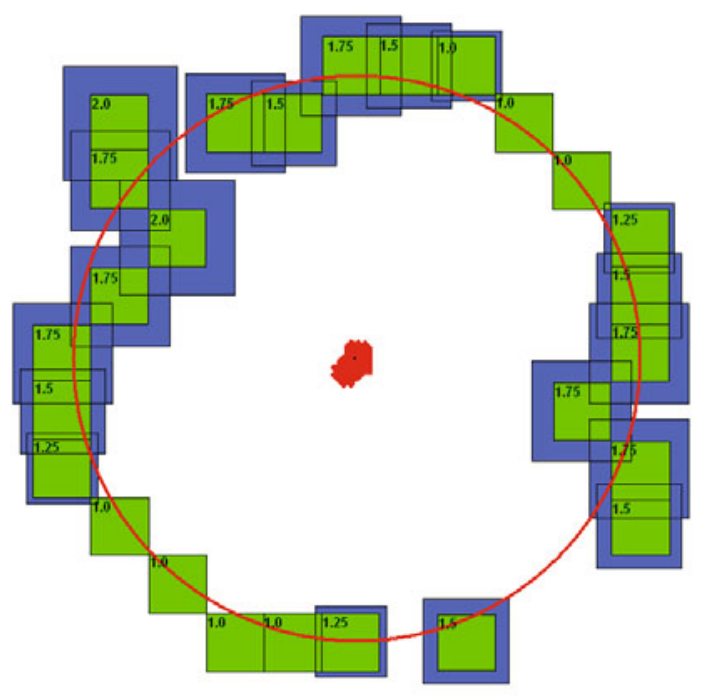

Fig. 4. Circle parameters exhaustive estimation with noisy data

\section{Generalized Reflection Symmetry and Biased Rotation Parameter Estimation}

As a second application, we show how the SGPB helps to visualize the errors that occur when noisy data are used in the rotation reconstruction algorithm recently proposed by Fontijne et al. [8]. For this we are going to introduce a generalization of the reflection symmetry.

\subsection{Bundles and Strips}

We define the line bundle $\mathbb{F}_{i}$ as the set of lines $\Delta$ that pass through $\mathbb{P}_{i}$ with slopes in the interval $\left[\operatorname{slope}\left(\mathbb{D}_{i}\right)\right.$, slope $\left.\left(\mathbb{D}_{i+1}\right)\right]$ (or $\left[\operatorname{slope}\left(\mathbb{D}_{i+1}\right)\right.$, slope $\left.\left(\mathbb{D}_{i+2}\right)\right]$ according to the values of $i$ ) as shown in the Figure 2(b). We also define a line strip $\operatorname{strip}\left(\mathbb{P}_{i}, \mathbb{P}_{j}\right)$ by the set of parallel lines bordered by $\Delta_{i} \in M$ passing through $\mathbb{P}_{i}$ for $i \in[1 ; 4]$ and $\Delta_{j} \in M$ passing through $\mathbb{P}_{j}$ for $j \in[5 ; 8]$ as shown in the figure $2(\mathrm{~b})$.

A line in the SGPB can either be characterized as a point inside the dual of the SGPB or by using bundles $\mathbb{F}_{i}$ and strips. Considering a line $D$ in the $S G P B$ two cases are possible. In the first case, the line $D$ passes through $\mathbb{P}_{i}$ then $D$ belongs to $\mathbb{F}_{i}$. In the second case the line $D$ does not pass through $\mathbb{P}_{i}$ then there exists $\mathbb{P}_{j}$ and $\mathbb{P}_{k}$ such that a translate $D^{\prime}$ of $D$ passes through $\mathbb{P}_{j}$ and another translate $D^{\prime \prime}$ of $D$ passes through $\mathbb{P}_{k}$. In this latter case, $D$ belongs to $\operatorname{strip}\left(\mathbb{P}_{j}, \mathbb{P}_{k}\right)$ (see Figure 2(b)). This leads to a second characterization of a line inside a SGPB: 
Property 1 (Lines in the SGPB). Every line in the SGPB belongs either to a bundle $\mathbb{F}_{i}$ or is included in a strip strip $\left(\mathbb{P}_{k}, \mathbb{P}_{l}\right)$

Furthermore, we have the additional property:

Property 2. The union of the 8 bundles covers the GPB.

Proof. Let $X$ a point of the GPB. Since the border of the GPB is composed of two convex polylines $\partial C_{1}$ and $\partial C_{2}$, there exists two lines $\Delta_{1}$ and $\Delta_{2}$ which pass through $X$ and respectively $p_{1} \in \partial C_{1}$ and $p_{2} \in \partial C_{2}$. Obviously, $p_{1}$ and $p_{2} \in \mathbb{P}_{i} . \square$

Generalized Reflection Symmetry for a pixel. When considering the perpendicular bisector $B$ of two points $p$ and $q$, it is obvious that $p$ is the image of $q$ and $q$ the image of $p$ through a reflection symmetry of axis $B$. Let us define the Generalized Reflection Symmetry $\operatorname{GRS}\left(P_{1}, P_{2}\right)$ of a point as the union of the reflection symmetries of axis the straight lines in the SGPB of two pixels $P_{1}$ and $P_{2}$. Not surprisingly, the image of a pixel $P_{3}$ by a $G R S\left(P_{1}, P_{2}\right)$ is not a pixel. Let us characterize the obtained region denoted $\mathcal{C}_{G R S\left(P_{1}, P_{2}\right)}\left(P_{3}\right)$ (See Figure 6).

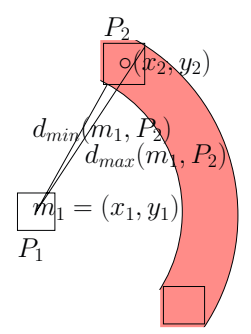

(a)

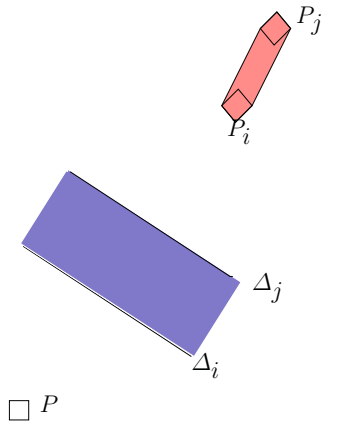

(b)

Fig. 5. (a) Image of the pixel $P_{2}$ by a partial bundle from $m_{1}$; (b) Image of the pixel $P$ by the $\operatorname{strip}\left(\Delta_{i}, \Delta_{j}\right)$

By construction, a point $p$ of $\mathcal{C}_{G R S\left(P_{1}, P_{2}\right)}\left(P_{3}\right)$ is the symmetric of a point $p_{3}$ belonging to the pixel $P_{3}$ with respect to a line $\Delta$ that belongs to $\operatorname{SGPB}\left(P_{1}, P_{2}\right)$. More formally, this can be written as:

$$
\mathcal{C}_{G R S\left(P_{1}, P_{2}\right)}\left(P_{3}\right)=\left\{p \in \mathbb{R}^{2} / \exists p_{3} \in P_{3}, \exists \Delta \in \operatorname{SGBP}\left(P_{1}, P_{2}\right), p=\operatorname{sym}\left(p_{3}, \Delta\right)\right\}
$$

where $\operatorname{sym}\left(p_{3}, \Delta\right)$ denotes the reflection symmetry of the point $p_{3}$ with respect to the line $\Delta$. Hence, the following characterization of a point belonging to $\mathcal{C}_{G R S\left(P_{1}, P_{2}\right)}\left(P_{3}\right)$ is obtained:

Definition 1 (GRS of a pixel). Let $P_{1}$ and $P_{2}$ be two pixels centered in $\left(x_{1}, y_{1}\right)$ and $\left(x_{2}, y_{2}\right)$ and the associated $S G P B\left(P_{1}, P_{2}\right)$. Let $\mathcal{C}_{G R S\left(P_{1}, P_{2}\right)}\left(P_{3}\right)$ be the image of the pixel $P_{3}$ by $G R S\left(P_{1}, P_{2}\right)$. A point $(x, y)$ belongs to $\mathcal{C}_{G R S\left(P_{1}, P_{2}\right)}\left(P_{3}\right)$ if the following assertions are true: 


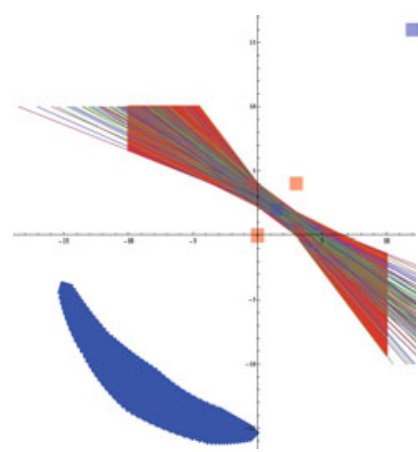

(a)

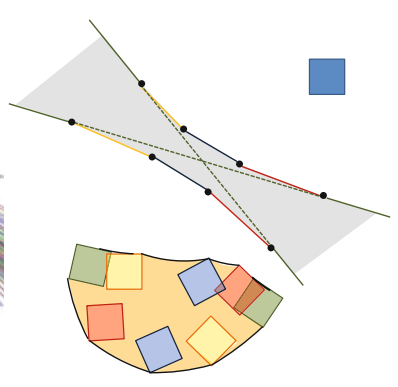

(b)

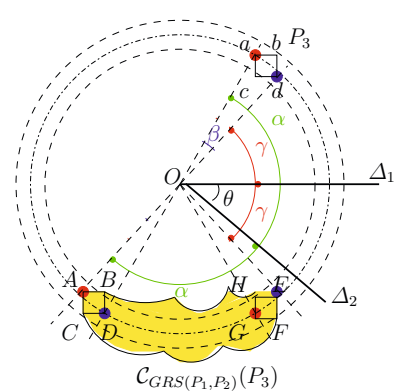

(c)

Fig. 6. (a) Generalized Reflection Symmetry of a pixel $(12,16)$ relatively to a SGPB between the pixel $(0,0)$ and $(3,4)$; (b) Image of a pixel by a GRS. The obtained shape is composed of ring pieces; (c) Symmetric of a pixel $P_{3}$ with respect to the lines $\Delta_{1}$ and $\Delta_{2}$.

(i) $\exists\left(x^{\prime}, y^{\prime}\right)$ such that $\left(x_{3}-0.5 \leq x^{\prime} \leq x_{3}+0.5\right)$ and $\left(y_{3}-0.5 \leq y^{\prime} \leq y_{3}+0.5\right)$

(ii) $(x, y)$ can be written as $\left(\frac{-2 a c-a^{2} x^{\prime}+b^{2} x^{\prime}-2 a b y^{\prime}}{a^{2}+b^{2}}, \frac{-2 b c-2 a b x^{\prime}+a^{2} y^{\prime}-b^{2} y^{\prime}}{a^{2}+b^{2}}\right)$ such that $\left(x_{2}, \frac{-c-a x_{2}}{b}\right),\left(x_{1}, \frac{-c-a x_{1}}{b}\right),\left(\frac{-b y_{1}-c}{a}, y_{1}\right),\left(\frac{-b y_{2}-c}{a}, y_{2}\right)$ satisfy the distance law (1).

Let us detail a way to construct the GRS of a pixel. The image of a pixel $P_{3}$ by a complet 1 line bundle passing through a point $p$ is a ring centered in $p$ defined by the circles centered in $p$ with radii $d_{\min }\left(p, P_{3}\right)$ and $d_{\max }\left(p, P_{3}\right)$. In our case, the bundle is not complete, the result is then a piece of ring (see Figure 5 (a)). Let $P_{i}$ and $P_{j}$ be the images of the pixel $P$ by the two lines which define a strip. The image of the pixel $P$ by this line strip is the strip bounded by the images of the pixel relatively to the line passing through the centers of $P_{i}$ and $P_{j}$ as shown in the Figure 5 (b).

Let $P_{3}$ be the pixel of which the image is to be computed. The lines $\mathbb{D}_{j}$ are the lines bounding the $\operatorname{SGPB}\left(P_{1}, P_{2}\right)$ and $\mathbb{P}_{i}$ the characteristic points of the $\operatorname{SGPB}\left(P_{1}, P_{2}\right)$. Let $I_{j}$ be the images of $P_{3}$ by $\mathbb{D}_{j}$. Since $\mathbb{D}_{1}=\mathbb{D}_{10}$ and $\mathbb{D}_{5}=\mathbb{D}_{6}$ (lemma 1), there are only eight different images. Then we consider the images of $P_{3}$ by the whole line bundle $\mathbb{F}_{i}$ that passes through $\mathbb{P}_{i}=\mathbb{D}_{l} \cap \mathbb{D}_{m}$. These images are the ring pieces starting with $I_{l}$, ending with $I_{m}$ of internal radius $d_{\min }\left(\mathbb{P}_{i}, P_{3}\right)$ and external radius $d_{\max }\left(\mathbb{P}_{i}, P_{3}\right)$. We therefore obtain eight ring pieces that form a closed object (since $\mathbb{D}_{1}=\mathbb{D}_{10}$ and $\mathbb{D}_{5}=\mathbb{D}_{6}$ ) as shown in the Figure 6(b).

The last building step consist in integrating the images of $P_{3}$ by all the possible strips that are included in the $\operatorname{SGPB}\left(P_{1}, P_{2}\right)$. This leads to a second characterization of the shape $\mathcal{C}_{G R S\left(P_{1}, P_{2}\right)}\left(P_{3}\right)$ :

\footnotetext{
${ }^{1}$ Complete means all the lines are passing through the point.

2 That means the slopes of the lines are included between slope $_{\min }$ and slope $_{\max }$.
} 
Characterization 1 (GRS of a pixel). A point belongs to the image of a pixel $P_{3}$ by the $G R S\left(P_{1}, P_{2}\right)$ between the pixel $P_{1}$ and $P_{2}$ if and only if it belongs to the set points defined by the eight pieces of rings centered in $\mathbb{P}_{i}$ for $1 \leq i \leq 4$ of radius $d_{\text {min }}\left(\mathbb{P}_{i_{1 \leq i \leq 4}}, P_{3}\right)$ and centered in $\mathbb{P}_{i}$ for $5 \leq i \leq 8$ of radius $d_{\text {max }}\left(\mathbb{P}_{i_{5 \leq i \leq 8}}, P_{3}\right)$, where the $\mathbb{P}_{i}$ are the characteristic points of the $S G B P$.

The angular arc between the extrema of the region (as defined below) $\mathcal{C}_{G R S\left(P_{1}, P_{2}\right)}\left(P_{3}\right)$ gives us an upper bound of "noise" that is added to a pixel when its image by a GRS is computed. Let consider the lines $\Delta_{1}$ and $\Delta_{2}$ that are the supports of the half-lines $\mathbb{D}_{1}$ and $\mathbb{D}_{5}$ (see Figure 6 (c)). They intersect at the point $O=\left(\frac{x_{1}+x_{2}}{2}, \frac{y_{1}+y_{2}}{2}\right)$. On Figure 6(c), the symmetric squares $A B D C$ and $E F G H$ of the square $a b d c$ by the lines $\Delta_{1}$ and $\Delta_{2}$ are the extrema of the region $\mathcal{C}_{G R S\left(P_{1}, P_{2}\right)}\left(P_{3}\right)$. As the lines $\Delta_{1}$ and $\Delta_{2}$ belong to the bundle of lines that pass through the point $O$, the squares $A B D C$ and $E F G H$ are contained in the ring centered in $O$ formed by the circles $\mathcal{C}_{1}$ and $\mathcal{C}_{2}$ of radii $R 1=d_{\text {min }}\left(O, P_{3}\right)$ and $R 2=d_{\max }\left(O, P_{3}\right)$. Since $B$ and $H$ belong to $\mathcal{C}_{1}$ and $C, F$ belong to $\mathcal{C}_{2}$, the points $A, D, G$ and $E$ belong to the circle centered in $O$ of radius $R 3=\frac{R 1+R 2}{2}$. Then, the angular length between the extrema of $\mathcal{C}_{G R S\left(P_{1}, P_{2}\right)}\left(P_{3}\right)$ is given by $(\beta+2 \theta)\left(\frac{R 1+R 2}{2}\right), \beta$ and $\theta$ respectively denote the angles between the lines $O a$ and $O d$ and the lines $\Delta_{1}$ and $\Delta_{2}$.

\subsection{Rotation Reconstruction Using the SGPB}

In this section, the rotation reconstruction algorithm proposed by Fontijne et al. 8] is adapted to noisy data using the $S G P B$. This algorithm is based on the Cartan-Dieudonné theorem [3] that decomposes $n D$ rotations into reflections. Methods based on this approach [2] need exact point correspondences and suffer from noisy data.

However, rotation reconstruction methods from point correspondences are used in many application domains such as Computer Vision or body movement analysis. Different approaches can be found in the literature [4179]. Their drawbacks are the impossibility to extend them in dimension higher than two or the subtle geometrical model and computations they need. A simple algorithm based on the decomposition of $n \mathrm{D}$ rotations into planar rotations has been developed by some of the authors and it has been shown to be rather robust against noisy data 1312 . But, contrary to the Fontijne et al. 8] algorithm, this approach is not well suited when the data are acquired incrementally.

The purpose of this section is to give some hints on how the SGPB could help to develop an adapted version of the Fontijne et al. [8] algorithm to noisy data. For this first attempt, only dimension two is explored but as SGPB is defined for any dimension, extension to $n \mathrm{D}$ is expected.

Rotation reconstruction algorithm. To reconstruct the rotation $R$ from $n$ points $p_{i}$ and their correspondences $p_{i}^{\prime}=R\left(p_{i}\right)$ the algorithm proposed in [8] works by finding successive reflections using the Cartan-Dieudonné theorem [3]. 


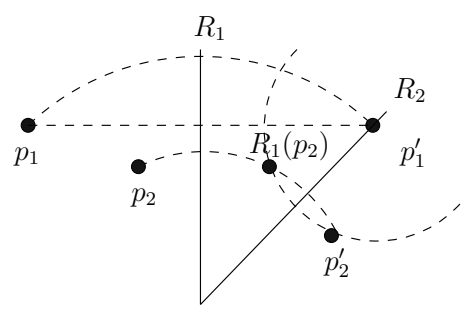

(a)

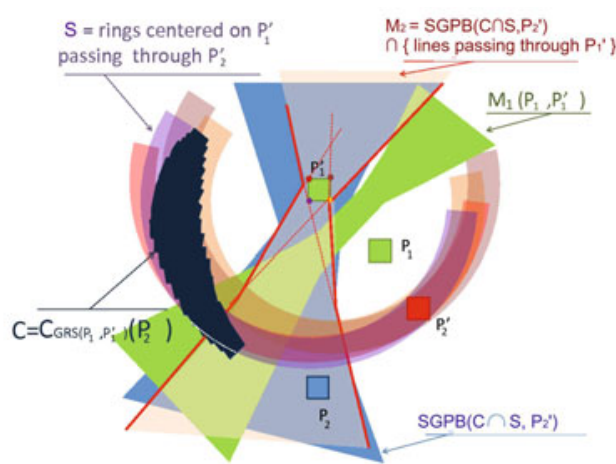

(b)

Fig. 7. (a) Reconstructing a 2D-rotation. Note that $p_{1}^{\prime}$ is contained in $R_{2}$, hence $p_{1}^{\prime}=$ $R_{2}\left(p_{1}^{\prime}\right)=R_{2}\left(R_{1}\left(p_{1}\right)\right)$. The rotation $R=R_{2}\left(R_{1}().\right)$ is a rotation about the intersection of $R_{1}$ and $R_{2}$. (b) Reconstruction of a noisy 2 D-rotation.

Hence, for each $i$, we have $p_{i}^{\prime}=R\left(p_{i}\right)=R_{k}\left(\cdots R_{2}\left(R_{1}\left(p_{i}\right)\right) \cdots\right)$ with $k=\left\lfloor\frac{n}{2}\right\rfloor$, where $R_{i}$ are the reflections 3 .

The $R_{i}$ are determined incrementally as perpendicular bisectors. The first reflection $R_{1}$ is determined as the perpendicular bisector of $p_{1}$ and $p_{1}^{\prime}$, the second reflection $R_{2}$ is the perpendicular bisector of $R_{1}\left(p_{2}\right)$ and $p_{2}^{\prime}$.

By construction, the reflections have the property that their composition does not move previously aligned points. For example, as

$$
p_{1}^{\prime}=R_{1}\left(p_{1}\right)=R\left(p_{1}\right)=R_{k}\left(\cdots R_{2}\left(R_{1}\left(p_{1}\right)\right) \cdots\right)
$$

we must have $R_{k}\left(\cdots R_{2}\left(p_{1}^{\prime}\right) \cdots\right)=p_{1}^{\prime}$.

The Figure 7 illustrates the algorithm in dimension two. It must be noted that $p_{1}^{\prime}=R_{1}\left(p_{1}\right)$ is on the line $R_{2}$ and it is the center of circle passing through $R_{1}\left(p_{2}\right)$ and $p_{2}^{\prime}$. Now, replacing the perpendicular bisectors with the SGPB could help to adapt the algorithm of Fontijne et al. [8] to noisy data.

Adaptation of the rotation reconstruction algorithm to noisy data. The algorithm described in the previous section supposes that exact point correspondences are provided. However, in the case of noisy data, the reflections became hard to determine. To deal with noisy data, the idea is to replace points by $n$ dimensional pixels and to introduce the SGPB into the reflection determination process. The expected result is a class of rotations that fit the input data.

Principle of the algorithm (see Figure $7(b)$ ). First the points $p_{i}$ and their corresponding points $p_{i}^{\prime}$ are replaced by $n$-dimensional pixels $P_{i}$ and $P_{i}^{\prime}$. Then, the SGPB $M_{1}=S G P B\left(P_{1}, P_{1}^{\prime}\right)$ (corresponding to $R_{1}$ in the exact case) is determined. Using $M_{1}$, the region $\mathcal{C}_{G R S\left(P_{1}, P_{1}^{\prime}\right)}\left(P_{2}\right)$ is computed. This region corresponds to $R_{1}\left(p_{2}\right)$ in the exact case.

${ }^{3}$ In this section, $R_{i}$ denotes indistinguishably the reflection and the line that determines the reflection. 
In order to restrict $\mathcal{C}_{G R S\left(P_{1}, P_{1}^{\prime}\right)}\left(P_{2}\right)$, we use the property that a circle centered on $p_{1}^{\prime}$ passing through $p_{2}^{\prime}$ and $R_{1}\left(p_{2}\right)$ exists (see Figure [7(a)). In the pixel case this means that we have to consider the set $S$ of all circles centered on points of $P_{1}^{\prime}$ passing through $P_{2}^{\prime}$. So region of interest for the next step is $\mathcal{C}_{G R S\left(P_{1}, P_{1}^{\prime}\right)}\left(P_{2}\right) \cap S$.

Now, the $\operatorname{SGBP}\left(\mathcal{C}_{G R S\left(P_{1}, P_{1}^{\prime}\right)}\left(P_{2}\right) \cap S, P_{2}^{\prime}\right)$ is computed (see Figure $\left.7(\mathrm{~b})\right)$. The obtained region must also be restricted with the set $L$ of lines that pass through $P_{1}^{\prime}$ while in the exact case the bisector $R_{2}$ passes through $p_{1}^{\prime}$ (see Figure [7(a)).

This finally determines the region $M_{2}=\operatorname{SGBP}\left(\mathcal{C}_{G R S\left(P_{1}, P_{1}^{\prime}\right)}\left(P_{2}\right) \cap S, P_{2}^{\prime}\right) \cap L$ corresponding $R_{2}$ in the exact case. In higher dimension, this process is continued with next point correspondences.

At the end of the algorithm we obtain a class of rotations that are determined by lines chosen in the sequence of the $M_{i}, 1 \leq i \leq n$.

At present, we only have experienced this process in dimension 2 and we face efficiency problems for computing the SGBP between a pixel and a complex region such as the $\mathcal{C}_{G R S\left(P_{1}, P_{1}^{\prime}\right)}\left(P_{2}\right)$. Currently, we are working on this efficiency problem and also on the extension of this algorithm to higher dimension.

\section{Conclusion}

In this paper, we studied the Simplified Generalized Perpendicular Bisector and its properties: whereas the classical Euclidean 2D perpendicular bisector (and all the commonly used medial axis notions), the SGPB of two regions is a $2 \mathrm{D}$ surface that collects all the perpendicular bisector of any couple of points of both regions. We have particularly defined the SGPB between two pixels of different sizes. The SGPB is useful to obtain an exhaustive parameter estimation of noisy circles. We have also characterized the lines in the SGPB by showing that the dual of a SGPB is a convex polygon and we have defined a new operation: the Generalized Reflection Symmetry which is a symmetry relatively to a SGPB. This operation allows us to adapt the algorithm of Fontijne et al. 8 to reconstruct rotations while taking noisy data into account. In future works, the GPB will be further studied. There are various notions of discrete bisector that have been proposed. They are, among other applications, used to analyze and filter medial axis [5]16 where the medial axis of a Jordan curve is in any point equidistant to its borders. These notions of discrete bisectors are discrete curves. How these notions are linked to the Generalized Bisector is one the question we would like to investigate. Especially the links with Voronoï diagrams, medial axis and skeletons seems promising. An extension in higher dimension will also be investigated.

\section{References}

1. Andres, E., Largeteau-Skapin, G., Rodríguez, M.: Generalized perpendicular bisector and exhaustive discrete circle recognition (2010) (submitted for publication at Graphical Models)

2. Aragon-Gonzalez, G., Aragon, J.L., Rodriguez-Andrade, M.A., Verde-Star, L.: Reflections, rotations, and pythagorean numbers, vol. 19, pp. 1-14 (2009) 
3. Audin, M.: Geometry, pp. 46-49. Springer, Heidelberg (2003)

4. Cheng, P.: Joint rotation between two attitudes in the spherical rotation coordinate system, vol. 37, pp. 1475-1482 (2004)

5. Couprie, M., Coeurjolly, D., Zrour, R.: Discrete bisector function and Euclidean skeleton in 2D and 3D. Image and Vision Computing 25(10), 1543-1556 (2007)

6. Dexet, M.: Architecture d'un modeleur discret à base topologique d'objets discret et méthodes de reconstruction en dimensions 2 et 3. Ph.D. thesis, Université de Poitiers (2006)

7. Farouki, R.T., Johnstone, J.K.: Computing point/curve and curve/curve bisectors. In: Fisher, R.B. (ed.) The Mathematics of Surfaces V, pp. 327-354. Oxford University, Oxford (1994)

8. Fontijne, D., Dorst, L.: Reconstructing rotations and rigid body motions from points correspondences as a sequence of reflections. In: Applied Geometric Algebras in Computer Science and Engineering, AGACSE 2010, Amsterdam, The Netherlands (June 14-16, 2010)

9. Gebken, C., Perwass, C., Sommer, G.: Parameter estimation from uncertain data in geometric algebra. Advances in Applied Clifford Algebra 18, 647-664 (2008)

10. Kerautret, B., Lachaud, J.-O.: Multi-scale analysis of discrete contours for unsupervised noise detection. In: Wiederhold, P., Barneva, R.P. (eds.) IWCIA 2009. LNCS, vol. 5852, pp. 187-200. Springer, Heidelberg (2009)

11. Peternell, M.: Geometric properties of bisector surfaces. Graphical Models 62(3), 202-236 (2000)

12. Richard, A., Fuchs, L., Andres, E., Largeteau-Skapin, G.: Decomposition of nDrotations: classification, properties and algorithm (2010) (submitted for publication at Graphical Models)

13. Richard, A., Fuchs, L., Charneau, S.: An algorithm to decompose $n$-dimensional rotations into planar rotations. In: Barneva, R., Brimkov, V., Hauptman, H., Natal Jorge, R., Tavares, J. (eds.) CompIMAGE 2010. LNCS, vol. 6026, pp. 60-71. Springer, Heidelberg (2010)

14. Rodríguez, M., Largeteau-Skapin, G., Andres, E.: Adaptive pixel resizing for multiscale recognition and reconstruction. In: Wiederhold, P., Barneva, R.P. (eds.) IWCIA 2009. LNCS, vol. 5852, pp. 252-265. Springer, Heidelberg (2009)

15. Rodríguez, M., Sere, A., Largeteau-Skapin, G., Andres, E.: Generalized perpendicular bisector and circumcenter. In: Barneva, R., Brimkov, V., Hauptman, H., Natal Jorge, R., Tavares, J. (eds.) CompIMAGE 2010. LNCS, vol. 6026, pp. 1-10. Springer, Heidelberg (2010)

16. Talbot, H., Vincent, L.: Euclidean skeletons and conditional bisectors. In: SPIE, vol. 1818, pp. 862-876 (1992)

17. Watson, G.: Computing Helmert transformations, vol. 197, pp. 387-394 (2006) 